\title{
Thermalization of interacting fermions and delocalization in Fock space
}

\author{
Clemens Neuenhahn and Florian Marquardt \\ Friedrich-Alexander-Universität Erlangen-Nürnberg, Institute for Theoretical Physics II, Staudtstrasse 7, 91058 Erlangen, Germany
}

(Received 14 September 2010; revised manuscript received 6 April 2012; published 1 June 2012)

\begin{abstract}
We investigate the onset of "eigenstate thermalization" and the crossover to ergodicity in a system of onedimensional fermions with increasing interaction. We analyze the fluctuations in the expectation values of most relevant few-body operators with respect to eigenstates. It turns out that these are intimately related to the inverse participation ratio of eigenstates displayed in the operator eigenbasis. Based on this observation, we find good evidence that eigenstate thermalization should set in even for vanishingly small perturbations in the thermodynamic limit.
\end{abstract}

DOI: 10.1103/PhysRevE.85.060101

PACS number(s): 05.30.-d, 02.30.Ik, 05.45.Mt, 05.70.Ln

Introduction. Statistical physics relies on the assumption that the system under investigation is in thermal equilibrium. However, what are the precise conditions for an isolated system to relax to thermal equilibrium? This question has a long history, including the groundbreaking observation by Fermi, Pasta, and Ulam [1] on an anharmonic chain of classical oscillators, where thermalization was not observed as expected [2]. Nowadays, thermalization in quantum many-body systems attracts a lot of theoretical attention [3], inspired by the new experimental possibilities in systems of cold atoms [4-6]. The trajectory of classical ergodic systems reaches all regions on the energy shell for sufficiently long times, establishing the microcanonical ensemble. Suitably chosen subsystems obey the Boltzmann distribution. In the quantum case, switching on interactions in a many-body system combines unperturbed eigenstates $|i\rangle$ of similar energies into new energy eigenstates: $|\alpha\rangle=\sum_{i} c_{i}^{\alpha}|i\rangle$. If the expectation values $A_{\alpha}=\langle\alpha|\hat{A}| \alpha\rangle$ of observables in these new eigenstates approach their microcanonical values $A_{\text {micro }}(E)$, as obtained by averaging over all unperturbed states in a small energy window around $E$, then the properties of thermal equilibrium are established in each many-body eigenstate. This is the idea behind the "eigenstate thermalization hypothesis" (ETH) [7,8] (see also Ref. [9]).

Recently, the ETH has been tested numerically [10-12] by means of exact diagonalization. For few-body observables such as the momentum distribution, indeed it was demonstrated that $A_{\alpha} \approx A_{\text {micro }}\left(E_{\alpha}\right)$ and that the fluctuations around $A_{\text {micro }}$ decrease with increasing interaction strength and system size.

Here, we investigate how fast thermal equilibrium, in the sense of ETH, is approached when increasing the system size. Our numerical results are obtained for a nonintegrable onedimensional (1D) model of interacting fermions that contains all the features of generic interacting systems. We primarily consider the momentum space occupation numbers $\hat{f}_{k}=\hat{c}_{k}^{\dagger} \hat{c}_{k}$ and the real-space single particle density matrix $\hat{\varrho}_{m}=\hat{c}_{1}^{\dagger} \hat{c}_{1+m}$.

We show that for sufficiently large interaction strength the fluctuations of expectation values $A_{\alpha}$ of these observables are determined by the inverse participation ratio (IPR) of eigenstates in the noninteracting Fock basis. Qualitatively, similar results were obtained recently $[13,14]$ and it was conjectured that the IPR might determine the deviations of steady state expectation values from the corresponding microcanonical value [15]. The IPR can be considered as the inverse number of Fock states contributing to $|\alpha\rangle$ (see, e.g., Refs. $[16,17])$ and thus keeps track of the delocalization of eigenstates in the many-body Fock space $[13,18]$.

We also consider an example of observables, which are not diagonal in the noninteracting basis. At large interactions, we observe a close connection between fluctuations and the IPR in the corresponding operator eigenbasis.

A crucially important question is how quickly fluctuations are suppressed upon increasing the system size, even for small interactions. We observe that for interactions $V$ larger than $\Delta_{f}$ (the mean level spacing between directly coupled Fock states) eigenstates delocalize in momentum Fock space $[19,20]$. Consistent with earlier analytical insights into Fockspace localization [21], the IPR drops exponentially with $V$ on a scale that depends on $\Delta_{f}$, which in turn decreases polynomially with system size. Combining this result with the observed IPR-fluctuation connection, we find strong evidence that fluctuations of the relevant observables considered here are suppressed to zero in the thermodynamic limit (TDL), establishing eigenstate thermalization, even for vanishingly small interactions. While this contrasts with recent observations on relaxation in a classical 1D system [22], it is consistent with the crucial assumption that tiny interactions are sufficient for thermalizing the system but can be neglected apart from that.

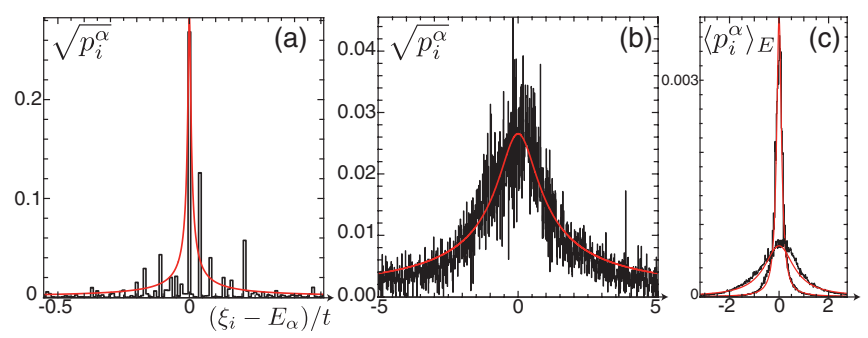

FIG. 1. (Color online) Probability distribution $p_{i}^{\alpha}=|\langle i \mid \alpha\rangle|^{2}$ of a many-body eigenstate $|\alpha\rangle$ in the noninteracting Fock basis (energies $\xi_{i}$ ). (a) For weak interaction $V / t=0.1$, the eigenstate is localized in Fock space, consisting of a few isolated peaks. (b) At large $V / t=1.3$, all Fock states with energies $\xi_{i}$ close to $E_{\alpha}$ contribute. (c) $p_{i}^{\alpha}$ averaged over a couple of nearby eigenstates in a range $\delta E / t=0.1$, for $V / t=$ $0.45,1.3$ (top, bottom). It can be approximated by a Lorentzian of width $\bar{\Gamma}$ (red/gray line). The energy was chosen to correspond to infinite effective temperature (see the main text). 
Model. We consider $n$ spinless 1D fermions with periodic boundary conditions on a lattice of $N$ sites and with a next-nearest-neighbor interaction breaking integrability. The Hamiltonian reads

$$
\begin{aligned}
\hat{H}_{0}+\hat{V}= & -t \sum_{i=1}^{N} \hat{c}_{i}^{\dagger} \hat{c}_{i+1}+\text { H.c. } \\
& +V \sum_{i=1}^{N}\left(\hat{n}_{i}-1 / 2\right)\left(\hat{n}_{i+2}-1 / 2\right) .
\end{aligned}
$$

The eigenstates $|i\rangle$ of $\hat{H}_{0}$ with $\xi_{i}=\langle i|\hat{H}| i\rangle$ are given by the Fock states of $n$ fermions in momentum space. Due to the translational symmetry, interactions do not mix states with different total momentum $K$. Therefore, each momentum sector $K$ with dimension $D_{K}$ will be considered separately. We exclude the $K=0$ sector as it possesses a trivial extra symmetry under reflection. In our numerical examples $n=7$, $N=21$, and $t=1$ sets the energy scale $(\hbar=1)$.

Fluctuations and IPR. In the following, we discuss the expectation values $f_{k}^{\alpha}$ of the momentum occupation numbers $\hat{f}_{k}=\hat{c}_{k}^{\dagger} \hat{c}_{k}$ (where $\hat{c}_{k} \equiv 1 / \sqrt{N} \sum_{j=1}^{N} e^{-i k x_{j}} \hat{c}_{j}$ ). Being interested in the properties of typical eigenstates, we analyze the statistics of an ensemble of states $|\alpha\rangle$ with similar eigenenergies $E_{\alpha} \in I_{E}=[E-\delta E, E+\delta E]$, which will be called in the following "eigenstate ensemble" (EE). The width of the energy window $\delta E$ has to be chosen to be small enough to avoid artifacts resulting from systematic dependencies on $E$. Averages with respect to the EE are denoted by $\langle\cdots\rangle_{E}$. For not too large interactions, one can easily show that $\left\langle f_{k}^{\alpha}\right\rangle_{E} \approx f_{k \text {,micro }}(E)$. However, the crucial statement of the ETH is that for each eigenstate $f_{k}^{\alpha} \rightarrow f_{k \text {,micro when going to }}$ the TDL, i.e., that the fluctuations of $f_{k}^{\alpha}$ from state to state vanish:

$$
\delta f_{k}^{2} \equiv\left\langle\left\{f_{k}^{\alpha}-\overline{f_{k}}\right\}^{2}\right\rangle_{E} \stackrel{N \rightarrow \infty}{\rightarrow} 0 .
$$

We introduced the EE variance $\delta f_{k}^{2}$ and $\overline{f_{k}}=\left\langle f_{k}^{\alpha}\right\rangle_{E}$. Representing $f_{k}^{\alpha}$ in the momentum Fock basis $f_{k}^{\alpha}=\sum_{i=1}^{D_{K}} p_{i}^{\alpha} f_{k}^{i}$ (with $f_{k}^{i}=\left\langle i\left|\hat{f}_{k}\right| i\right\rangle$ and $p_{i}^{\alpha} \equiv|\langle i \mid \alpha\rangle|^{2}$ ) this statement becomes plausible. For strong interaction, typical eigenstates are spread out widely in Fock space [Fig. 1(b)], i.e., they are composed of a large number of Fock states close in energy. Due to the law of large numbers, we thus expect the fluctuations to decay as the mean inverse number of Fock states contributing to $|\alpha\rangle$, i.e., as the mean IPR

$$
\chi=\left\langle\sum_{i=1}^{D_{K}}\left(p_{i}^{\alpha}\right)^{2}\right\rangle_{E}
$$

Before deriving the connection between $\delta f_{k}^{2}$ and $\chi$ formally, we focus on the numerical results for the present model. Figure 2 shows $\delta f_{k}^{2}$ as a function of $V$ evaluated at various energies. The eigenenergies can be reexpressed in terms of effective temperatures $T$, with $E_{T} \equiv \operatorname{tr}_{K}\left(\hat{H} e^{-\hat{H} / T}\right) / \operatorname{tr}_{K}\left(e^{-\hat{H} / T}\right)$. The results are compared to the IPR, or more precisely to the sum over the variances $\operatorname{Var}_{E}\left(p_{i}^{\alpha}\right)=\left\langle\left(p_{i}^{\alpha}\right)^{2}\right\rangle_{E}-\left\langle p_{i}^{\alpha}\right\rangle_{E}^{2}$ (see below), demonstrating that indeed $\delta f_{k}^{2} \propto \sum_{i} \operatorname{Var}_{E}\left(p_{i}^{\alpha}\right)$ even for small interactions. This is in stark contrast to the case of integrability conserving nearest-neighbor interaction (see the

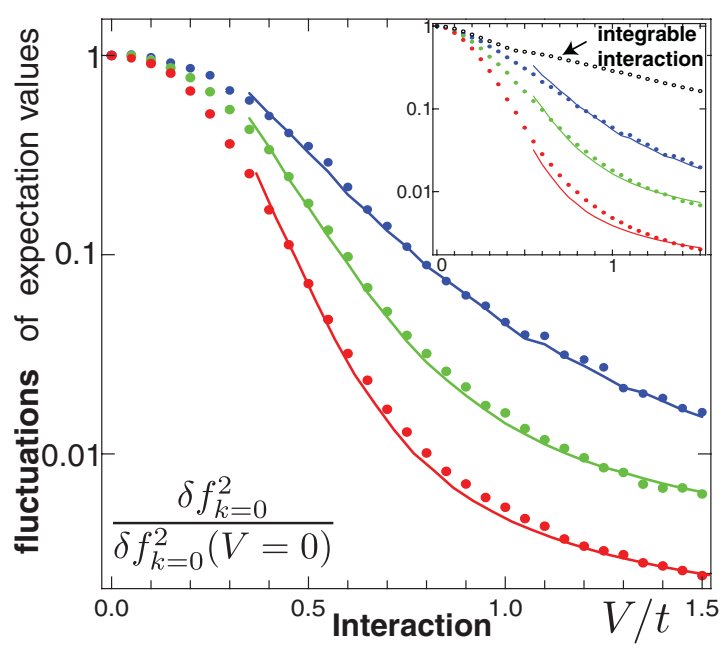

FIG. 2. (Color online) Fluctuations of expected occupation number $\left\langle\alpha\left|\hat{f}_{k}\right| \alpha\right\rangle$ between eigenstates decrease with increasing interaction strength $V$, indicating eigenstate thermalization. The plot shows the variance $\delta f_{k=0}^{2}$, for states with an effective temperature $T / t=$ $1.4,2.1, \infty$ (from top to bottom), with energy shells of width $\delta E / t=$ 0.25 . Solid lines show const $\times \bar{f}_{k=0}\left(1-\bar{f}_{k=0}\right) \sum_{i} \operatorname{Var}_{E}\left(p_{i}^{\alpha}\right)$, with a slightly $T$-dependent constant. Finally, the results are averaged over all total momentum sectors. Inset: As in the main figure, but with $\delta f_{k}^{2}$ averaged over all $k$. Black, open dots show $\delta f_{k}^{2}$ averaged over all $k$ for an integrable model with nearest-neighbor interactions [at $T=\infty$ and $K /(2 \pi / N)=1]$. $K$ averages are only performed to improve statistics. The same results are obtained for individual $K$ sectors.

inset in Fig. 2), where the suppression of $\delta f_{k}^{2}$ with $V$ is much smaller than in the prior case.

Formally, representing $\delta f_{k}^{2}$ in terms of $p_{i}^{\alpha}$, one finds

$$
\delta f_{k}^{2} \simeq \overline{f_{k}}\left(1-\overline{f_{k}}\right) \sum_{i} \operatorname{Var}_{E}\left(p_{i}^{\alpha}\right)+\sum_{i \neq j}^{D_{K}} \delta f_{k}^{i j} \operatorname{Cov}_{E}\left(p_{i}^{\alpha} p_{j}^{\alpha}\right),
$$

with $\delta f_{k}^{i j}=\left(f_{k}^{i} f_{k}^{j}-{\overline{f_{k}}}^{2}\right)$ and the covariance matrix $\operatorname{Cov}_{E}\left(p_{i}^{\alpha} p_{j}^{\alpha}\right) \equiv\left\langle p_{i}^{\alpha} p_{j}^{\alpha}\right\rangle_{E}-\left\langle p_{i}^{\alpha}\right\rangle_{E}\left\langle p_{j}^{\alpha}\right\rangle_{E}$. The first term in Eq. (4) contains the suppression of $\delta f_{k}^{2}$ with an increasing number of Fock states contributing to a typical eigenstate. It is essentially determined by $\chi$ [we note that $\chi \approx \sum_{i} \operatorname{Var}_{E}\left(p_{i}^{\alpha}\right)$ below the ergodic regime (see below)]. We replaced $\sum_{i}\left(f_{k}^{i}-\right.$ $\left.\bar{f}_{k}^{2}\right) \operatorname{Var}_{E}\left(p_{i}^{\alpha}\right) \rightarrow\left(\bar{f}_{k}-\bar{f}_{k}^{2}\right) \sum_{i} \operatorname{Var}_{E}\left(p_{i}^{\alpha}\right)$, which is justified as $\operatorname{Var}_{E}\left(p_{i}^{\alpha}\right)$ is a smooth function of $i$. The prefactor $\overline{f_{k}}\left(1-\overline{f_{k}}\right)$ represents the variance of the momentum occupation numbers for the noninteracting case.

The off-diagonal contributions in Eq. (4) are sensitive to residual correlations within eigenstates and are expected to become small for strong perturbations. Surprisingly, for strong enough interactions, they approximately reproduce the diagonal part of Eq. (4). Thus, even though $\delta f_{k}^{2}$ is still determined by the IPR, one observes a deviation of the prefactor of $O(1)$. A very similar observation was made in Ref. [23] while investigating finite fermionic systems with random two-body interactions and was traced back to the strong correlations between matrix elements of two-body interaction matrices. 
To sum up, we find that the fluctuations in the expectation value of $\hat{f}_{k}$ from eigenstate to eigenstate are determined by the IPR $\chi$. Thus, in the following, we discuss how $\chi$ decreases with increasing $V$ and system size.

Definitions. For the discussion of $\chi$, we set up a few technical definitions. We introduce the effective density $\rho_{f}^{i}(\omega)$ of Fock states $|j\rangle$ coupling to a state $|i\rangle$ of energy $\xi_{i} \in$ $I_{E}$ (i.e., $\langle i|\hat{V}| j\rangle \neq 0$ ), where the energy difference between both states is $\xi_{i}-\xi_{j}=\omega$. Averaging over a couple of states $|i\rangle$ (indicated by $\langle\cdots\rangle_{E}^{0}=\left[\sum_{i, \xi_{i} \in I_{E}}\right]^{-1}\left[\sum_{i, \xi_{i} \in I_{E}} \cdots\right]$ ) one obtains the mean effective density of states $\rho_{f}(\omega, E)=$ $\left\langle\rho_{f}^{i}(\omega)\right\rangle_{E}^{0}$. In the following, the density of interacting states close in energy $(\omega \simeq 0)$ will be denoted by $\rho_{f}=\Delta_{f}^{-1}$. Furthermore, we define the mean squared matrix element $\overline{V^{2}}=\frac{\Delta_{f}}{\delta \omega} \int_{-\delta \omega / 2}^{\delta \omega / 2} d \omega^{\prime}\left\langle\sum_{j=1 ; i \neq j}^{D_{K}} V_{i j}^{2} \delta\left(\xi_{j}-\xi_{i}-\omega^{\prime}\right)\right\rangle_{E}^{0}$. Numerically, both are evaluated in the limit of small interactions, where $\xi_{j} \simeq\left\langle j\left|\hat{H}_{0}\right| j\right\rangle$.

Discussion of IPR. For $\sqrt{\overline{V^{2}}} \ll \rho_{f}^{-1}$, in general, eigenstates can be obtained within perturbation theory. A given momentum Fock state gets perturbed by the set of directly coupling states, and eigenstates consist of a small number of sharp peaks [Fig. 1(a)], i.e., they are localized in Fock space. Increasing the coupling strength $\sqrt{\overline{V^{2}}} \sim \rho_{f}^{-1}$, one enters the regime of delocalized eigenstates [20]. Perturbation theory breaks down and the IPR starts to decrease rapidly [see Fig. 3(a)]. In this regime, the fluctuations $\delta f_{k}^{2}$ become directly determined by $\chi$. Surprisingly, one observes an exponential decay of $\chi$ and we

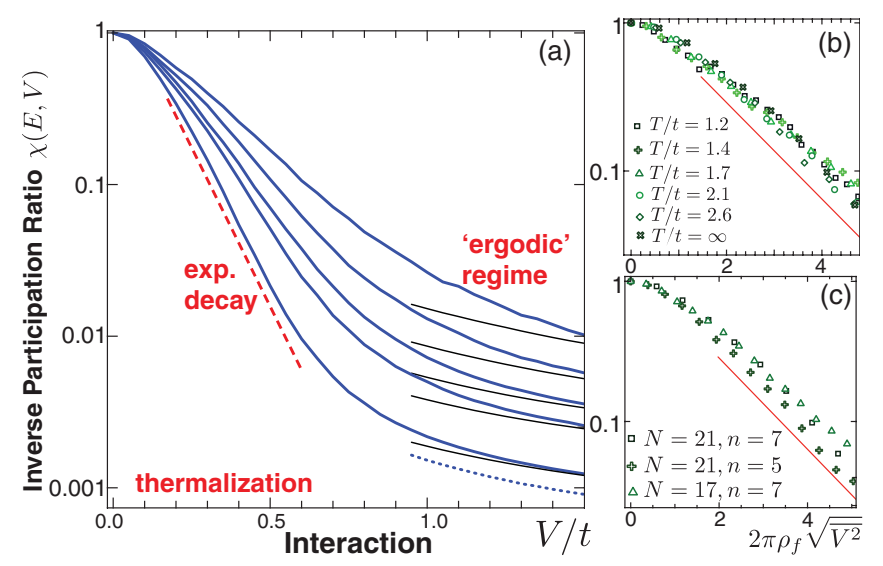

FIG. 3. (Color online) (a) The inverse participation ratio $\chi$ decreases as many-body eigenstates get more delocalized for increasing interaction strength $V$. From top to bottom: Effective temperatures $T / t=1.4,1.7,2.1,2.6, \infty$ (thick, blue lines; averaged over all momentum sectors). The most important feature is an exponential decay at intermediate interactions [red dashed line; see Eq. (5)] followed by a power-law tail in the "ergodic" regime (see the main text), where it can be well approximated by Eq. (6) (shown only for $T=\infty$ and $K=2 \pi / N$ : blue dotted line). In the ergodic regime, the amplitudes $c_{i}^{\alpha}$ are Gaussian distributed, leading to $\chi \rightarrow 3 \sum_{i}\left\langle p_{i}^{\alpha}\right\rangle_{E}^{2}$ (thin, black lines). (b) "Scaling plot": As before, but plotted vs $2 \pi \rho_{f} \sqrt{\overline{V^{2}}}$ (and only for a single $K$ ). (c) Similar plot, but for various system sizes, at $T / t=\infty$. The red line displays Eq. (5), with $\mathcal{C} \approx 0.75$, for comparison. found good numerical evidence that

$$
\chi \propto \exp \left\{-\mathcal{C} \rho_{f} \sqrt{\overline{V^{2}}}\right\}
$$

The numerical constant $\mathcal{C}$ is independent of temperature and system size. In Figs. 3(b) and 3(c), the IPR is shown as a function of the scaling variable $2 \pi \rho_{f} \sqrt{\overline{V^{2}}}$ for eigenstates at different energies $E$ and for various $N$ and $n$. Indeed, in good accordance to Eq. (5), all curves collapse to the same scaling curve. An explanation of this exponential decay of $\chi$ might be found in the two-particle nature of the interaction. In Ref. [21] it was argued (in a random matrix setting) that, for moderate interaction strength, typical eigenstates are composed of independent pairs of interacting fermions. Eigenstates decompose into direct products of Fock-state pairs, resulting in an exponential decay of $\chi$, of the form given by Eq. (5). While this exponential decay (and additional corrections) was confirmed numerically for a random quantum dot Hamiltonian [16], here we find it in a translationally invariant system without disorder.

We now discuss the dependence on system size. The effective density of states $\rho_{f}(\omega)$ scales as $N^{3}$. For example, at large $T$, we have $\rho_{f}(\omega) t \simeq N^{3} \rho^{2}(1-\rho)^{2} r(\omega)$, with the density $\rho$. For our model, $r(\omega) \propto \ln (t / \omega)$ for $\omega \rightarrow 0$ due to transitions of particle pairs around the inflection point of the $-2 t \cos (k)$ dispersion, resulting in $\rho_{f}^{-1} \propto t /\left(N^{3} \ln N\right)$ (assuming a cutoff scale $\omega / t \sim 1 / N$ ). Together with the scaling of the matrix elements $\sqrt{\overline{V^{2}}}=v(E) V / N$, this would yield $\chi \propto \exp \left\{-\tilde{\mathcal{C}} N^{2} \ln N \rho^{2}(1-\rho)^{2} V / t\right\}$, with $\tilde{\mathcal{C}}$ being independent of $N$.

According to this estimate, at any fixed, but arbitrarily small interaction strength, thermalization will occur in the TDL. This crucial result corroborates general physical expectations. We stress that consequently thermalization does not require interactions comparable to finite thresholds such as the single particle bandwidth.

For completeness, we now discuss the regime of such large interactions $V \sim t$. This is a regime of "ergodic" eigenstates, by which we understand states which in principle are composed of all momentum Fock states with given $K$ close in energy [cf. Fig. 1(b)]. No Fock states are excluded a priori, e.g., due to the two-body nature of $\hat{V}$ or further symmetries from contributing to an ergodic eigenstate. The amplitudes $c_{i}^{\alpha}$ become Gaussian distributed random variables [7,8] (for further information see Ref. [24]) with a Lorentzian variance $[7,25,26]\left\langle p_{i}^{\alpha}\right\rangle_{E} \simeq \frac{1}{\pi \rho_{K}} \frac{\bar{\Gamma}}{\left(\xi_{i}-E-\bar{\delta}\right)^{2}+\bar{\Gamma}^{2}}$, where $\rho_{K}$ denotes the full many-body density of states for total momentum $K$, scaling as $(N-1) ! /(N-n) ! n !$. We checked that in this regime the nearest-neighbor level spacing statistics agrees with the Gaussian orthogonal ensemble (GOE)-Wigner surmise, characteristic for quantum chaotic systems. Due to the Gaussian distribution for $c_{i}^{\alpha}$, one finds $\chi=3 \sum_{i}\left\langle p_{i}^{\alpha}\right\rangle_{E}^{2}$, resulting in

$$
\chi \simeq 3 /\left(2 \pi \bar{\Gamma} \rho_{K}\right)
$$

which is in fairly good agreement with the numerical results in Fig. 3(a), demonstrating the suppression of $\delta f_{k}^{2}$ by the inverse density of states as it was conjectured in Refs. $[7,8]$. The width 

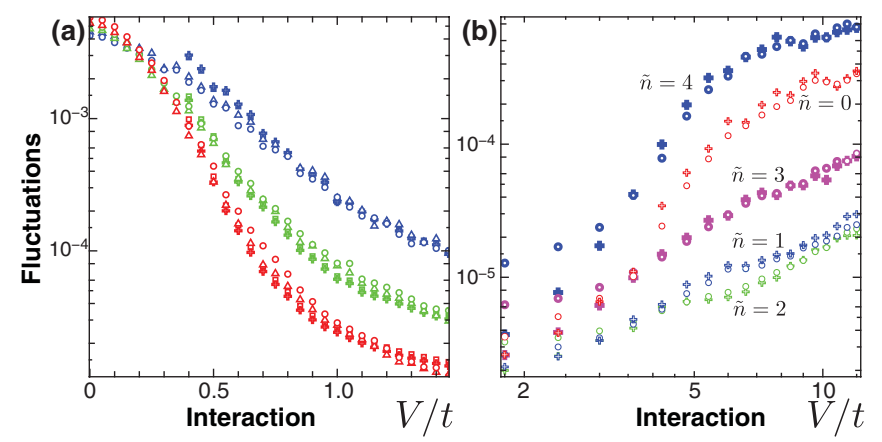

FIG. 4. (Color online) (a) Fluctuations of the single particle density matrix $\varrho_{m}^{\alpha}$ for EEs at $T / t=1.4,2.1, \infty$ [top to bottom; blue (upper), green (middle), red (lower): Real and imaginary parts are considered separately $(\Delta, \circ)]$. These are compared to const $\times$ $\left(\overline{\operatorname{Re} \varrho_{m}^{2}}-{\overline{\operatorname{Re} \varrho_{m}}}^{2}\right) \sum_{j} \operatorname{Var}_{E}\left(p_{j}^{\alpha}\right) \quad$ (crosses) with $\overline{\operatorname{Re} \varrho_{m}}, \overline{\operatorname{Re} \varrho_{m}^{2}}=$ $\sum_{j}\left[\operatorname{Re} \varrho_{m}^{j},\left(\operatorname{Re} \varrho_{m}^{j}\right)^{2}\right]\left\langle p_{j}^{\alpha}\right\rangle_{E}$ and the analogous expression for the imaginary part $(\square)$. Finally, the results are averaged over all $m \neq 0$. One finds good agreement for large enough $V$. (b) Fluctuations of $C_{m}^{\alpha}$ averaged over all $m \neq 0$ excluding the special cases $m=2, N-2$ (see the main text). As $V \gg t$, the density of states splits into bands. We consider various eigenstate ensembles lying each in the center of a single band [labeled by the mean number of interacting pairs $\tilde{n}=0, \ldots, 4$ : red (thin), blue (thin), green (thin), magenta (thick), blue (thick) crosses]. This is compared to const $\times \overline{C_{m}^{2}} \sum_{J} \operatorname{Var}_{E}\left(p_{J}^{\alpha}\right)$ (dots) averaged in the same fashion. One finds good agreement for $V / t \gg 1$. In (a), (b), $K=4 \pi / N$ and constants depend on $T$ and $\tilde{n}$, respectively.

$\bar{\Gamma}$ can be extracted numerically [24]. For small $V$, Fermi's golden rule applies and $\bar{\Gamma} \propto V^{2} / t$.

Eventually, for very large $V \gg t$, interactions dominate and the density of states splits into bands. Whether integrability is restored in this limit has to be analyzed carefully, but is beyond the scope and interest of our analysis.

Other observables. The crucial IPR-fluctuation connection holds for further observables, e.g., the operator $\hat{c}_{1}^{\dagger} \hat{c}_{1+m}$ corresponding to the single particle density matrix $\varrho_{m}^{\alpha} \equiv$ $\left\langle\alpha\left|\hat{c}_{1}^{\dagger} \hat{c}_{1+m}\right| \alpha\right\rangle$ is diagonal in the momentum Fock basis for a given $K$. In fact, $\varrho_{m}^{\alpha}=N^{-1} \sum_{k} e^{i k m} f_{k}^{\alpha}$. We consider both the fluctuations of the real and imaginary part of $\varrho_{m}^{\alpha}$ within the EE and find [Fig. 4(a)] that for large enough $V, \delta \operatorname{Re} \varrho_{m}^{2}$ and $\delta \operatorname{Im} \varrho_{m}^{2}$ [defined analogously to Eq. (2)] are $\propto \sum_{j} \operatorname{Var}_{E}\left(p_{j}^{\alpha}\right)$.

In contrast, the operator $\hat{n}_{1} \hat{n}_{1+m}$ is diagonal in the realspace Fock basis $|J\rangle$ (for a given $K$ ). Nevertheless, for $V \gg t$ one observes [Fig. 4(b)] that the fluctuations of $C_{m}^{\alpha} \equiv\left\langle\alpha\left|\hat{n}_{1} \hat{n}_{1+m}\right| \alpha\right\rangle$, i.e., $\delta C_{m}^{2} \equiv\left\langle\left(C_{m}^{\alpha}\right)^{2}\right\rangle_{E}-\left\langle\left(C_{m}^{\alpha}\right)\right\rangle_{E}^{2}$, become $\propto \overline{C_{m}^{2}} \sum_{J} \operatorname{Var}_{E}\left(p_{J}^{\alpha}\right)$ with $p_{J}^{\alpha} \equiv|\langle\alpha \mid J\rangle|^{2}$ and $\overline{C_{m}^{2}} \equiv$ $\sum_{J}\left(C_{m}^{J}\right)^{2}\left\langle p_{J}^{\alpha}\right\rangle_{E}$. In this limit, the density of states splits into bands centered around $\tilde{n} V+V(N / 4-n)$, where $\tilde{n}$ denotes the number of interacting pairs. In each band, the fluctuations increase with increasing $V$. The cases $m=2, N-2$ are special, as we are dealing with next-nearest-neighbor interactions and periodic boundary conditions. The fluctuations of $C_{2}^{\alpha}$ and $C_{N-2}^{\alpha}$ are largely suppressed for $V / t \gg 1$ and not longer proportional to $\sum_{J} \operatorname{Var}_{E}\left(p_{J}^{\alpha}\right)$.

Conclusions. We investigated the interaction induced onset of eigenstate thermalization in a system of 1D fermions. We found that the fluctuations of the expectation value of the momentum occupation number and the single particle density matrix from state to state are proportional to the IPR of eigenstates displayed in the momentum space Fock basis. For small interactions the latter decays exponentially. The interaction scale for the onset of this decay is determined by the mean level spacing between directly coupled Fock states and vanishes in the thermodynamic limit. This corroborates the physical expectation that in this limit thermalization sets in at arbitrarily small interactions.

Acknowledgments. We thank P. G. Silvestrov, F. Göhmann, and A. Polkovnikov for fruitful discussions related to this work. Financial support by DIP, NIM, the Emmy-Noether program, and the SFB/TR 12 is gratefully acknowledged.
[1] E. Fermi, J. Pasta, and S. Ulam, Document LA-1940, 1955.

[2] J. Ford, Phys. Rep. 213, 271 (1992).

[3] A. Polkovnikov, K. Sengupta, A. Silva, and M. Vengalattore, Rev. Mod. Phys. 83, 863 (2011).

[4] M. Greiner, O. Mandel, T. W. Hänsch, and I. Bloch, Nature (London) 419, 51 (2002).

[5] T. Kinoshita, T. Wenger, and D. S. Weiss, Nature (London) 440, 900 (2006).

[6] S. Hofferberth, I. Lesanovsky, B. Fischer, T. Schumm, and J. Schmiedmayer, Nature (London) 449, 324 (2007).

[7] J. M. Deutsch, Phys. Rev. A 43, 2046 (1991).

[8] M. Srednicki, Phys. Rev. E 50, 888 (1994).

[9] M. Rigol and M. Srednicki, Phys. Rev. Lett. 108, 110601 (2012).

[10] M. Rigol, V. Dunjko, and M. Olshanii, Nature (London) 452, 854 (2008).

[11] M. Rigol, Phys. Rev. Lett. 103, 100403 (2009).

[12] M. Rigol, Phys. Rev. A 80, 053607 (2009).

[13] L. F. Santos and M. Rigol, Phys. Rev. E 81, 036206 (2010).

[14] E. Canovi, D. Rossini, R. Fazio, G. E. Santoro, and A. Silva, Phys. Rev. B 83, 094431 (2011).
[15] V. A. Yurovsky and M. Olshanii, Phys. Rev. Lett. 106, 025303 (2011).

[16] X. Leyronas, P. G. Silvestrov, and C. W. J. Beenakker, Phys. Rev. Lett. 84, 3414 (2000).

[17] N. Linden, S. Popescu, A. J. Short, and A. Winter, Phys. Rev. E 79, 061103 (2009).

[18] P. G. Silvestrov, Phys. Rev. Lett. 79, 3994 (1997).

[19] B. L. Altshuler, Y. Gefen, A. Kamenev, and L. S. Levitov, Phys. Rev. Lett. 78, 2803 (1997).

[20] B. Georgeot and D. L. Shepelyansky, Phys. Rev. Lett. 79, 4365 (1997).

[21] P. G. Silvestrov, Phys. Rev. E 58, 5629 (1998).

[22] A. C. Cassidy, D. Mason, V. Dunjko, and M. Olshanii, Phys. Rev. Lett. 102, 025302 (2009).

[23] V. V. Flambaum, G. F. Gribakin, and F. M. Izrailev, Phys. Rev. E 53, 5729 (1996).

[24] See Supplemental Material at http://link.aps.org/supplemental/ 10.1103/PhysRevE.85.060101 for details.

[25] B. Lauritzen, P. F. Bortignon, R. A. Broglia, and V. G. Zelevinsky, Phys. Rev. Lett. 74, 5190 (1995).

[26] V. V. Flambaum and F. M. Izrailev, Phys. Rev. E 61, 2539 (2000). 